\title{
Impact of Restrictive Intravenous Fluid Replacement and Combined Epidural Analgesia on Perioperative Volume Balance and Renal Function Within a Fast Track Program ${ }^{1}$
}

\author{
Martin Hübner, M.D., ${ }^{*} \ddagger$ Markus Schäfer, M.D., ${ }^{* \dagger}$ Nicolas Demartines, M.D., ${ }^{*}{ }^{*}, 3$ Sven Müller, M.D., ${ }^{*}$ \\ Konrad Maurer, M.D. $\dagger$ †erner Baulig, M.D., $\dagger$ Pierre A. Clavien, M.D., Ph.D., ${ }^{*}$ Marco P. Zalunardo, M.D., $\dagger$ \\ and Zurich Fast Track Study Group ${ }^{\dagger}$ \\ *Department of Visceral and Transplantation Surgery; $\dagger$ Institute of Anaesthesiology, University Hospital Zurich, Zurich, Switzerland; \\ and $\ddagger$ Department of Visceral Surgery, University Hospital CHUV, Lausanne, Switzerland
}

Originally submitted June 9, 2010; accepted for publication August 27, 2010

Background and Objective. Key factors of Fast Track (FT) programs are fluid restriction and epidural analgesia (EDA). We aimed to challenge the preconception that the combination of fluid restriction and EDA might induce hypotension and renal dysfunction.

Methods. A recent randomized trial (NCT00556790) showed reduced complications after colectomy in FT patients compared with standard care (SC). Patients with an effective EDA were compared with regard to hemodynamics and renal function.

Results. 61/76 FT patients and 59/75 patients in the SC group had an effective EDA. Both groups were comparable regarding demographics and surgery-related characteristics. FT patients received significantly less i.v. fluids intraoperatively $(1900 \mathrm{~mL}$ [range 1100-4100] versus $2900 \mathrm{~mL}$ [1600-5900], $P<0.0001)$ and postoperatively (700 mL [400-1500] versus $2300 \mathrm{~mL}$ [1800-3800], $P<0.0001)$. Intraoperatively, 30 FT compared with 19 SC patients needed colloids or vasopressors, but this was statistically not significant $(P=0.066)$. Postoperative requirements were low in both groups (3 versus 5 patients; $P=0.487$ ). Pre- and postoperative values for creatinine, hematocrit, sodium, and potassium were similar, and no patient developed renal dysfunction in either group. Only one of 82 patients having an EDA without a bladder catheter had urinary retention. Overall, FT patients had fewer postoperative complications (6 versus 20 patients; $P=0.002)$ and a shorter

\footnotetext{
${ }^{1}$ PODIUM presentation (S63) at the 2009 ASCRS Annual Meeting, May 2-6, 2009, Hollywood, Florida.

${ }^{2}$ Listed at the end of the article.

${ }^{3}$ To whom correspondence and reprint requests should be addressed at Department of Visceral Surgery, University Hospital, Lausanne, Rue du Bugnon 46,1011 Lausanne, Switzerland. E-mail: demartines@chuv.ch.
}

median hospital stay (5 [2-30] versus 9 d [6-30]; $P<$ 0.0001) compared with the SC group.

Conclusions. Fluid restriction and EDA in FT programs are not associated with clinically relevant hemodynamic instability or renal dysfunction. $\odot 2012$ Elsevier Inc. All rights reserved.

Key Words: enhanced recovery; fast track; epidural analgesia; fluid restriction; hemodynamics; renal function.

\section{INTRODUCTION}

Optimal fluid management represents a key issue of perioperative care of patients undergoing major abdominal surgery. The successful advent of fast track (FT) programs has provided new aspects to the ongoing debate of perioperative fluid management [1-6].

Traditional fluid management aims to maintain blood pressure and heart rate in order to prevent hypovolemiainduced chances of microcirculation that may be associated with organ dysfunction [6, 7]. As a consequence, patients generally have a postoperative fluid overload that is reflected by a significant weight gain $[1,6,8]$.

So called FT concepts or enhanced recovery after surgery (ERAS) programs have been increasingly implemented into clinical practice during recent years. By limiting patients' perioperative stress response, postoperative complication rates and length of hospital stay can be reduced [2, 3, 5]. FT programs are primarily based on the use of epidural analgesia (EDA) to minimize opioid consumption, restrictive perioperative fluid management, early postoperative oral nutrition, and early ambulation $[2,3,5,9,10]$. 
The beneficial effects of thoracic EDA on pain reduction, pulmonary complications, and postoperative intestinal motility have largely been demonstrated [11-19]; drawbacks are transient arterial hypotension in about $10 \%$, pruritus and urinary complications [14, 18-21]. However, the impact of a restrictive fluid management on organ function is still under debate, and opponents are concerned for hypovolemia-induced organ dysfunction like renal insufficiency, myocardial ischemia, and impaired wound healing [6,7]. On the other hand, excessive perioperative fluid administration may exert deleterious effects on cardiopulmonary function, and prolong postoperative bowel arrest [1, 6, 22-25]. In fact, there is increasing evidence in the literature that restrictive fluid regimens are favorable to reduce cardiopulmonary complications and enhance postoperative recovery without compromising wound healing [1, 3, 5, 6, 9, 22, 23, 25, 26].

We were recently able demonstrate that patients undergoing elective open colonic surgery have a significantly reduced complication rate, if they were included in a FT program [27]. The two independent predictors for low postoperative complications were an efficient EDA and perioperative fluid restriction [27]. As outlined before, restrictive fluid management and EDA may both lead individually to arterial hypotension, renal dysfunction, and electrolyte disturbance. Their combined impact has not yet been examined in detail. Since this multicentric prospective randomized trial provided precise data on different organ function, we performed for the present study a post hoc analysis in patients with an efficient EDA.

We aimed to assess whether additional fluid restriction had a negative impact on preservation of hemodynamics and renal function in patients having an effective EDA. Furthermore, electrolyte disturbances as well as clinical outcome were separately assessed.

\section{METHODS}

A prospective randomized trial (NCT00556790) assessing a FT regimen versus standard care (SC) was performed in 156 patients undergoing open elective colon resection at four surgical departments in Switzerland (Fig. 1) [27].

Patients in the SC group received a fixed restricted fluid regime according to institutional guidelines that were based on established recommendations [6, 28]. They received Ringer's lactate at $2 \mathrm{~mL}$ per $\mathrm{kg}$ bodyweight per $\mathrm{h}$ for preoperative loading, and $10 \mathrm{~mL}$ per $\mathrm{kg}$ bodyweight per $h$ during the surgery, respectively. The actual bodyweight was measured usually the day before surgery. In the FT group, preoperative fasting (nil per mouth) time was 2-6 h for clear liquids. Loading volume to compensate external and internal loss caused by preoperative fasting, vasodilatation, and epidural analgesia (pre-block hydration) was performed by using Ringer's lactate solution at $1 \mathrm{~mL}$ per $\mathrm{kg}$ bodyweight per $\mathrm{h}$. Intraoperatively, crystalloid fluid administration was limited to $5 \mathrm{~mL}$ per $\mathrm{kg}$ bodyweight per h. Preoperative fluid loading according to the reported formulas was initiated upon entry in the operation room area. The epidural catheter was habitually placed before induction of general anesthesia; therefore, fluid loading was administered approximately 30-45 min prior to use of the EDA and for a total time of about $45-60 \mathrm{~min}$ before skin incision. Intravenous fluid administration in the FT group was discontinued at postoperative $\mathrm{d} 1$, unless there were medical indications to do otherwise. In the SC group, patients received $2000 \mathrm{~mL}$ Ringer's lactate per $24 \mathrm{~h}$ until postoperative $\mathrm{d} 3$.

Additional colloid fluids or low-dose vasopressors were given, when mean arterial pressure dropped permanently ( $>1 \mathrm{~h}$ or three consecutive measurements) below $60 \mathrm{~mm} \mathrm{Hg}$ or urine output was $(3 \times)$ lower than $0.5 \mathrm{~mL} / \mathrm{kg} / \mathrm{h}$. Vasopressors were privileged in order to avoid fluid overload. Low-dose vasopressors were norepinephrine up to $5 \mu \mathrm{g} / \mathrm{min}$ or ephedrine at a bolus dose of 10-25 mg intravenously (maximum $150 \mathrm{mg} / 24 \mathrm{~h}$ ). Blood transfusion was limited to a hematocrit $<25 \%$. Patients in the FT group were allowed to drink immediately after surgery and to resume an oral diet on postoperative $d 1$, while the SC group started drinking and oral nutrition on postoperative $d 2$ and full oral nutrition on postoperative $d 4$. An epidural catheter with ropivacaine $0.33 \%$ or bupivacaine $0.25 \%$ was placed at thoracic level $6-9$ preoperatively and removed on postoperative $d 2$. For additional analgesia, paracetamol was given intravenously at a fixed rate $(4 \times 1 \mathrm{~g} / \mathrm{d})$. A failure of EDA (inefficient EDA) was defined by the need for additional intravenous opioids. Perioperative fluid administration was recorded for the first $24 \mathrm{~h}$ after surgery. At postoperative d 1 , the urinary catheter was removed according to the study protocol.

Outcome of primary interest in the present study was perioperative vasopressor requirements and/or need for additional fluid administration indicating clinically relevant hemodynamic instability. Of note, patient's outcome was assessed in this study by a per protocol analysis; as we were interested in the effect of EDA with and without additional fluid restriction, we included only patients with an efficient EDA in the present subgroup analysis. Secondary outcome parameters included plasma concentrations of sodium, potassium, creatinine, and hematocrit values pre- and postoperatively that served as surrogate parameters for perioperative fluid shifts. Furthermore, perioperative creatinine values were used to assess for risk of renal dysfunction according to the AKIN classification system (RIFLE criteria) [29]. Postoperative complications (30-d morbidity) were graded according to its severity, and a validated therapy-orientated complication score on a five-point scale described was used [30].

Statistical analysis was performed using standard software package SPSS 14.0 (SPSS, Inc., Chicago, IL). Descriptive statistics are expressed as mean [ \pm standard deviation] or median [range] as appropriate. The Mann-Whitney U test was used to compare continuous variables, Chi-square and Fischer's exact tests were used for comparison of discrete variables. $P$-value $<0.05$ was considered statistically significant.

\section{RESULTS}

Overall, 156 patients were included in the study and randomly assigned to either $\mathrm{FT}(n=78)$ or $\mathrm{SC}(n=78)$. There were two patients in the FT group and three patients in the SC group who were excluded due to withdrawal or other protocol violation. Another 31 patients (FT: 15, SC: 16) were excluded in the present study for EDA failure. Finally, 61 out of 76 FT patients (80\%) and 59 out of 75 SC patients (79\%) had an effective EDA and were included in the present per protocol analysis (Fig. 1).

\section{Patients' Characteristics}

The FT and SC groups matched well regarding gender, body mass index, American Society of Anesthetists 


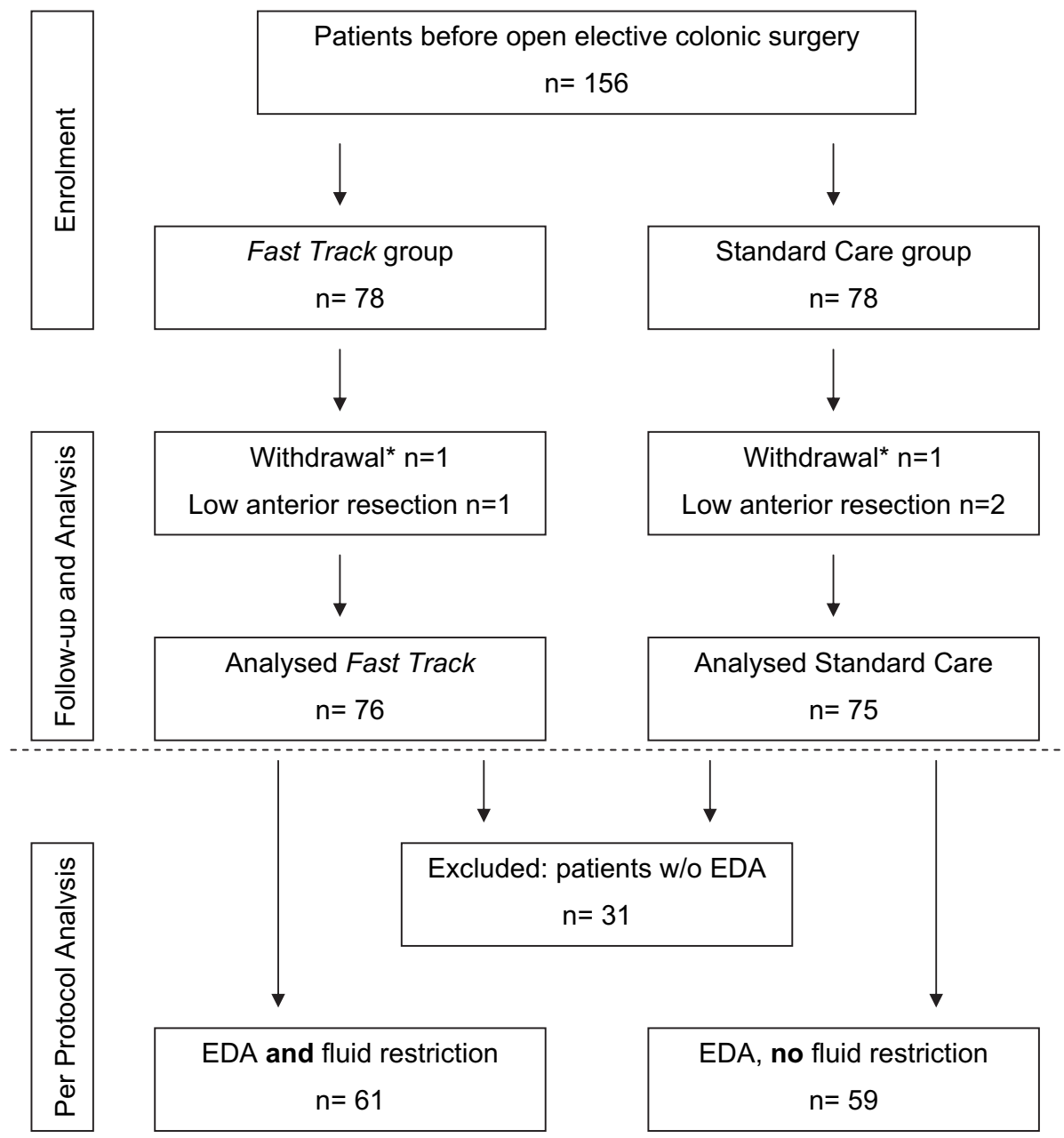

FIG. 1. Study flow chart. Patient flow of NCT00556790. For assessment of the combined effect of epidural analgesia (EDA) and fluid restriction on hemodynamics, a per protocol analysis was performed (below dotted line). The final analysis included 120 patients with a functional EDA; 61 of them had additionally a perioperative fluid restriction (Fast Track), while 59 had a traditional fluid management (Standard Care).

(ASA) class, indication for surgery, type and duration of the operation, as shown in Table 1. Patients in the FT group were slightly older (median of 62 y versus 59 in the SC group, $P=0.037$ ). The vast majority of patients underwent colon resection for cancer (92\% FT group, $85 \%$ SC group). Median operative times were 130 and 110 min for the FT group and SC group, whereby the difference was not statistically different. Median intraoperative blood loss was similar with $280 \mathrm{~mL}(0-1100$ $\mathrm{mL})$ in the FT group and $250 \mathrm{~mL}(0-1350 \mathrm{ml})$ in the $\mathrm{SC}$ group $(P=0.544)$.

Intraoperative and Postoperative Intravenous Fluid Replacement (Table 2, Fig. 2)

By adhering to the study protocol, FT patients received significantly less crystalloids intraoperatively compared to SC patients (median volume: FT group $1850 \mathrm{~mL}$ versus SC group $2900 \mathrm{~mL} ; P<0.0001)$. There were more FT patients who needed additional treatment by using colloids and/or vasopressors compared with the SC group to maintain intraoperative blood pressure and urinary output (FT group 30 of 61 patients, $49 \%$ versus SC group 19 of 59 patients, $32 \% ; P=0.066$ ). Overall intraoperative fluid replacement was predominantly determined by crystalloid application.

During the postoperative course, the restrictive intravenous fluid regime was maintained in the FT group; hence, median fluid volume needed for FT patients was only $700 \mathrm{~mL}$. SC patients received 2250 $\mathrm{mL}$, and the difference was statistically significant $(P<0.0001)$. Overall, perioperative intravenous fluid replacement was 2600 and $4950 \mathrm{~mL}$ for FT patients and SC patients, respectively $(P<0.0001)$. Of note, FT patient were allowed to start drinking immediately after the operation. Only three of $61 \mathrm{FT}$ patients and five of 59 SC patients $(P=0.487)$ needed a low dose vasopressor regimen to maintain mean arterial pressure and urine output, postoperatively. Two FT and two SC patients needed perioperative blood transfusions. 


\section{TABLE 1}

Patient Demographics and Operation Characteristics

\begin{tabular}{lccc}
\hline & FT & SC & $P$ \\
\hline No. of patients & 61 & 59 & \\
Gender (F/M) & $41 / 20$ & $49 / 10$ & 0.472 \\
Age (y) & $62(27-91)$ & $59(39-89)$ & 0.037 \\
ASA 1+2/3+4 & $41 / 20$ & $47 / 12$ & 0.150 \\
BMI $\left(\mathrm{kg} / \mathrm{m}^{2}\right)$ & $26(18-33)$ & $25(19-35)$ & 0.981 \\
Indication: malignant/benign & $56 / 5$ & $50 / 9$ & 0.266 \\
Left/right-sided resection & $40 / 21$ & $39 / 20$ & 1.000 \\
Operating time (min) & $130(60-210)$ & $110(55-230)$ & 0.112 \\
Blood loss (mL) & $280(0-1100)$ & $250(0-1350)$ & 0.544 \\
\hline
\end{tabular}

Values are median (range) for age, BMI, operating time, and blood loss.

$\mathrm{FT}=$ fast track $; \mathrm{SC}=$ standard care $; \mathrm{ASA}$ score = American Society of Anesthetists; BMI = body mass index.

"Left" includes left hemicolectomy and rectosigmoid resection, "Right" includes right hemicolectomy and transverse resections.

"Statistical significance $(P<0.050)$.

\section{Electrolyte Balance and Renal Function}

Pre- and postoperative creatinine levels did not differ significantly and remained within the limits considered physiologic in both patient groups. No patient in either group had a creatinine increase of more than $10 \%$ postoperatively. Furthermore, no patient developed acute renal failure up to $30 \mathrm{~d}$ after surgery. Postoperative hematocrit values were slightly but significantly reduced in the SC group (median 38 versus $35 \%, P<0.0001$ ). Pre- and postoperative sodium levels were similar in FT patients $(136.7 \pm 3.6 \mathrm{mmol} / \mathrm{L}$ versus $136.4 \pm$ $2.6 \mathrm{mmol} / \mathrm{L})$ compared with the SC group $(138.0 \pm 3.6$ $\mathrm{mmol} / \mathrm{L}$ versus $136.9 \pm 2.8 \mathrm{mmol} / \mathrm{L}$ ); potassium levels showed likewise only little variation (FT: $4.4 \pm 0.5$ $\mathrm{mmol} / \mathrm{L}$ versus $4.3 \pm 0.4 \mathrm{mmol} / \mathrm{L} ; \mathrm{SC}: 4.3 \pm 0.7 \mathrm{mmol} / \mathrm{L}$ versus $4.1 \pm 0.5 \mathrm{mmol} / \mathrm{L}$ ). Changes of creatinine and hematocrit expressed in median delta values (postoperative-preoperative value) showed significantly higher postoperative creatinine increase and hematocrit decrease in the FT group compared with SC group indicating relative intravascular hypovolemia (Fig. 3).

\section{Complication Rates and Hospital Stay}

Overall postoperative morbidity was $10 \%$ (6 of 61 patients) in the FT group compared with $34 \%$ (20 of 59 patients) in SC patients; and the difference was statistically significant $(P=0.002)$. Grades III and IV complications were two bleeding complications (FT: 1, SC: 1), two pulmonary embolisms (FT: 1, SC: 1 ), and one anastomotic leak (SC). Median hospital stay was significantly reduced in the FT group compared with the SC group with $5 \mathrm{~d}$ [2-30] versus $9 \mathrm{~d}[6-30](P<0.0001)$, respectively (Fig. 4).

According to the study protocol, urinary catheters were removed the morning of postoperative $d 1$ in 82 patients of both groups with a functioning EDA with only two complications: one urinary retention needing reinsertion of a catheter and one urinary tract infection. Comparatively, there was one urinary infection in the remaining 38 patients having their bladder catheter disconnected only after removal of the EDA.

\section{DISCUSSION}

Fluid overload negatively affects outcome after major abdominal surgery and has to be considered versus the risk of hypovolemic hypotension induced by overly restrictive fluid regimens [3, 6, 28]. Since enhanced recovery program recommend additional use of thoracic EDA, its sympathetic block might trouble hemodynamics even further [14, 18-21]. To assess the combined effect of these two measures, patients undergoing open colon resection within an enhanced recovery program have been analyzed in this present post hoc subgroup analysis [27].

We found that $30 \%$ and $10 \%$ of all patients had an intraoperative and postoperative arterial hypotension or reduced urinary output, respectively. A stringent fluid management had no additional significant adverse effect in patients with an EDA. Our findings confirm the analysis of Marret et al. that postoperative arterial hypotension is observed in approximately $10 \%$ of patients with EDA undergoing colorectal surgery [20].

TABLE 2

Intra- and Postoperative Intravenous Fluid Administration

\begin{tabular}{lccc}
\hline & FT & SC & \\
\hline No. of patients & 61 & 59 & \\
Intra-OP crystalloids $(\mathrm{mL})^{*}$ & $1850(1100-3800)$ & $2900(1600-4900)$ & $<0.0001$ \\
Post-OP crystalloids $(\mathrm{mL})^{*}$ & $700(400-1500)$ & $2250(1800-3800)$ & $<0.0001$ \\
24 h fluids $(\mathrm{mL})^{*}$ & $2600(1800-4900)$ & $4950(3900-9700)$ & $<0.0001$ \\
\hline
\end{tabular}

Values are median and range in brackets for intravenous fluids $(\mathrm{mL})$.

$\mathrm{FT}=$ fast track; $\mathrm{SC}=$ standard care.

Intra-OP $=$ intraoperative; Post-OP $=$ postoperative.

*Statistical significance $(P<0.050)$. 


\section{A Intra-OP hemodynamic support}

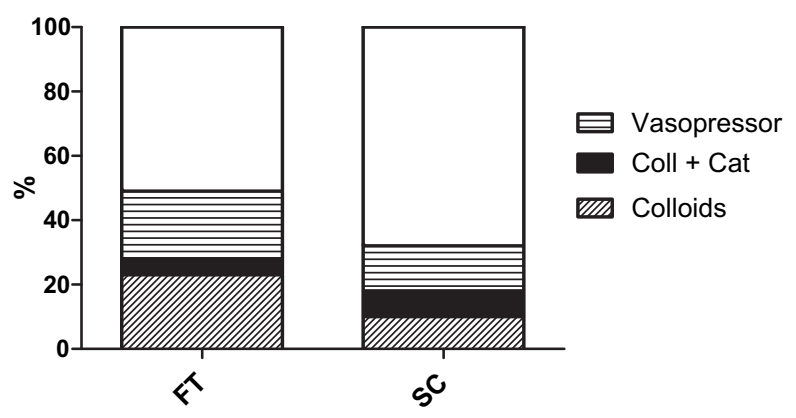

B Post-OP hemodynamic support

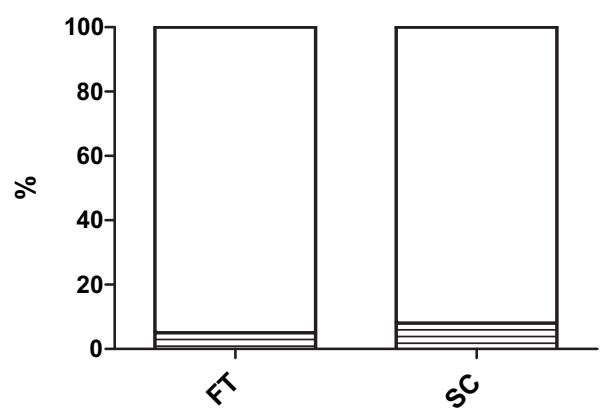

FIG. 2. Intra- and postoperative hemodynamic support. (A) Percentage of patients with intraoperative (intra-OP) need for colloids (shaded slope), vasopressors (shaded horizontally) or both (black). (B) Percentage of patients with postoperative (post-OP) need for vasopressors (shaded horizontally). $\mathrm{FT}=$ fast track; $\mathrm{SC}=$ standard care.

Arterial hypotension was countered in all patients preferentially with vasopressors rather than crystalloid infusions in order to avoid further postoperative fluid overload, which has been shown to affect outcome after major abdominal surgery adversely [6, 31]. Indeed, patients with additional fluid restriction in the FT group had significantly reduced complications and shorter hospital stay. Several prospective clinical trials, including this study, have shown that avoidance of fluid overload in combination or without a FT program primarily decreases nonsurgical complications and disturbance of wound healing [1, 6, 8, 26, 27, 32]. It was found that crystalloid fluids had negatively affected cardiopulmonary function in healthy volunteers [33]. Measures to minimize a preoperative hypovolemic state by reducing preoperative fasting to a minimum for example, or avoiding perioperative hypovolemia seem necessary in FT programs [3, 5, 10, 23].

Alternatively to a stringent fluid regime, some groups propagate a goal directed plasma volume expanding fluid administration controlled by esophagus Doppler or pulse contour analyzed cardiac index [6, 7, 34, 35]. However, its clinical applicability is demanding and the benefit of these work-intensive algorithms and invasive tools compared with simple dogmatic
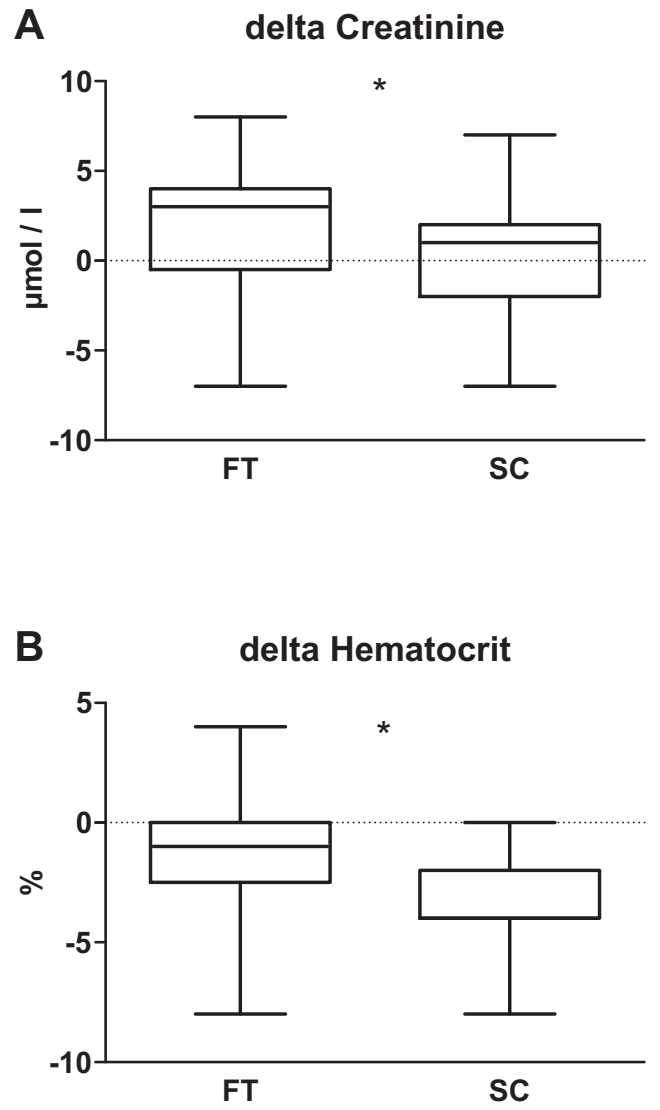

FIG. 3. Delta values for creatinine and hematocrit. Box plots with minimal and maximal differences (postoperative-preoperative) for creatinine (A) and hematocrit $(\mathrm{B}) . \mathrm{FT}=$ fast track; $\mathrm{SC}=$ standard care. ${ }^{*}$ Statistical significance $(P<0.050)$.

restrictive crystalloid based fluid regimes in colonic surgery has not been shown yet.

FT patients showed a significantly higher increase in their postoperative creatinine level compared with the SC group. However, no patient in either group appeared to be at risk of renal dysfunction according to the AKIN classification system (RIFLE criteria), as none showed a creatinine increase of more than $10 \%$ postoperatively in either group [29]. Furthermore, the absolute difference of only $2.0 \mu \mathrm{mol} / \mathrm{L}$ may not be clinically relevant, but may rather reflect a dilution effect of the liberal compared with the restrictive fluid management. This assumption is supported by a slightly more pronounced decrease of the postoperative hematocrit value in the SC group despite similar blood loss. Finally, no postoperative renal failure occurred up to 30 d after surgery.

Since an EDA at the thoracic level 6-9 without a urinary catheter was not associated with higher urinary retention rate, our results suggest that prolonged urinary catheterization is not necessary. These findings are confirmed by a prospective study by Basse et al. [36]. 
A Patients with complications

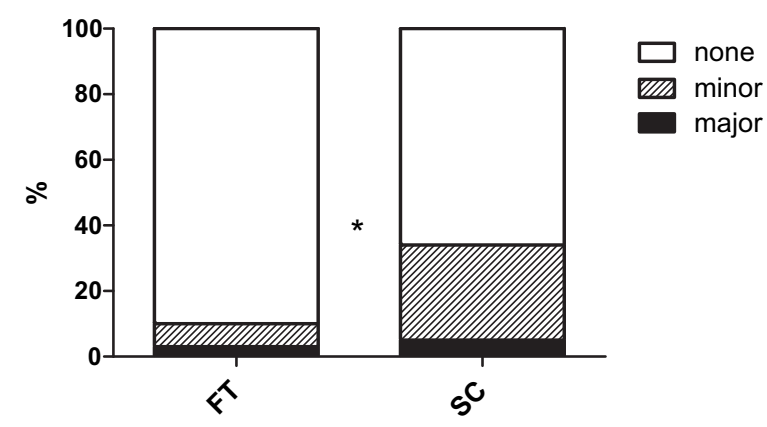

B Hospital stay

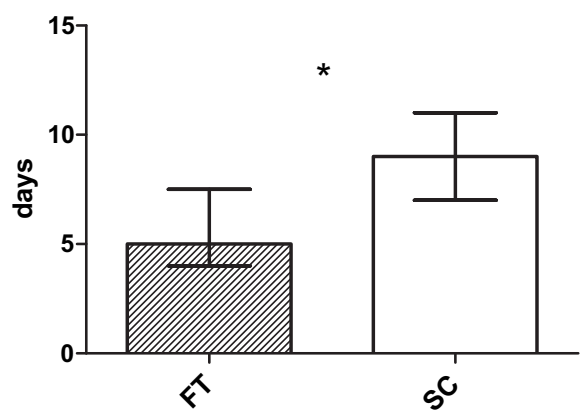

FIG. 4. Clinical outcome measures. (A) Percentage of patients with minor (shaded) and major (black) complications. Major complication is defined as $\geq 3 \mathrm{~b}$ according to [30]. (B) Median hospital stay with interquartile range (error bars). FT = fast track; SC: standard care. *Indicates statistical significance $(P<0.050)$.

Several issues of the present study need to be discussed. First, randomized data should usually be analyzed according to the intention-to-treat principle to obtain results that are realistic and generalizable. Therefore, intention-to-treat analysis was performed to compare complications and hospital stay between FT and SC patients [27]. However, a per protocol analysis gives a more accurate estimate of perioperative hemodynamic instability in patients with an effective EDA with different fluid replacement regimens; therefore, the present analysis was performed per protocol. Further, the sample size was calculated for an assumed reduction of postoperative complications [27], and might be insufficient to control type II error regarding the outcome measures addressed in this study. However, 120 patients constitute a fair number in a field, where randomized data are still pretty scarce.

In conclusion, avoiding perioperative fluid overload and an effective EDA are key factors in decreasing postoperative complications within an enhanced recovery program. Adding a stringent fluid regimen to a functional EDA appears to have only marginal impact on hemodynamic stability and renal function being of little clinical relevance.

\section{ACKNOWLEDGMENTS}

MH, SM, PAC, and ND have received research support and/or lecture fees from Fresenius-Kabi, Switzerland.

Contributors for the Zurich Fast Track Trial Group are as follows: Sven Müller, Martin Hübner, Nicolas Demartines, Department of Visceral Surgery, CHUV, Lausanne and formerly Visceral and Transplant Surgery, University Hospital Zurich; Pierre A. Clavien, Visceral and Transplant Surgery, University Hospital Zurich; Marco P. Zalunardo, Konrad Maurer, Baulig Werner, Institute of Anesthesiology, University Hospital Zurich; Marco Decurtins, Department of Surgery, Kantonsspital Winterthur; Lukas Eisner, Department of Surgery, Kantonsspital Olten; Italo Castelli, Department of Anesthesiology, Kantonsspital Olten; Hans P. Keller, Department of Anesthesiology, Kantonsspital Winterthur; Igor Langer, Department of Visceral Surgery, CHUV, Lausanne; Hans Gelpke, Felix Grieder, Department of Surgery, Kantonsspital Winterthur; Thorsten Carstensen, Andreas Gehrz, Department of Surgery, Kantonsspital Olten; Thierry Spahr, Institute of Anesthesiology, University Hospital Zurich; Reto Paganoni, Department of Anesthesiology, Kantonsspital Olten.

\section{REFERENGES}

1. Brandstrup B, Tonnesen H, Beier-Holgersen R, et al. Effects of intravenous fluid restriction on postoperative complications: Comparison of two perioperative fluid regimens: A randomized assessor-blinded multicenter trial. Ann Surg 2003;238:641.

2. Fearon KC, Ljungqvist O, Von Meyenfeldt M, et al. Enhanced recovery after surgery: A consensus review of clinical care for patients undergoing colonic resection. Clin Nutr 2005;24:466.

3. Lassen K, Soop M, Nygren J, et al. Consensus review of optimal perioperative care in colorectal surgery: Enhanced Recovery After Surgery (ERAS) Group recommendations. Arch Surg 2009;144:961.

4. Lobo DN. Fluid overload and surgical outcome: Another piece in the jigsaw. Ann Surg 2009;249:186.

5. Wind J, Polle SW, Fung Kon Jin PH, et al. Systematic review of enhanced recovery programmes in colonic surgery. Br J Surg 2006;93:800.

6. Rahbari NN, Zimmermann JB, Schmidt T, et al. Meta-analysis of standard, restrictive and supplemental fluid administration in colorectal surgery. Br J Surg 2009;96:331.

7. Gan TJ, Soppitt A, Maroof M, et al. Goal-directed intraoperative fluid administration reduces length of hospital stay after major surgery. Anesthesiology 2002;97:820.

8. Lobo DN, Bostock KA, Neal KR, et al. Effect of salt and water balance on recovery of gastrointestinal function after elective colonic resection: A randomised controlled trial. Lancet 2002; 359:1812.

9. Kehlet H, Wilmore DW. Evidence-based surgical care and the evolution of fast-track surgery. Ann Surg 2008;248:189.

10. Weimann A, Braga M, Harsanyi L, et al. ESPEN Guidelines on Enteral Nutrition: Surgery including organ transplantation. Clin Nutr 2006;25:224.

11. Ahlers O, Nachtigall I, Lenze J, et al. Intraoperative thoracic epidural anaesthesia attenuates stress-induced immunosuppression in patients undergoing major abdominal surgery. $\mathrm{Br} \mathrm{J}$ Anaesth 2008;101:781.

12. Ali M, Winter DC, Hanly AM, et al. Prospective, randomized, controlled trial of thoracic epidural or patient-controlled opiate analgesia on perioperative quality of life. Br J Anaesth 104:292.

13. Carli F, Mayo N, Klubien K, et al. Epidural analgesia enhances functional exercise capacity and health-related quality of life after colonic surgery: Results of a randomized trial. Anesthesiology 2002;97:540.

14. Cashman JN, Dolin SJ. Respiratory and haemodynamic effects of acute postoperative pain management: Evidence from published data. Br J Anaesth 2004;93:212. 
15. Guay J. The benefits of adding epidural analgesia to general anesthesia: A meta-analysis. J Anesth 2006;20:335.

16. Hanna MN, Murphy JD, Kumar K, et al. Regional techniques and outcome: What is the evidence? Curr Opin Anaesthesiol 2009;22:672.

17. Kehlet H. Epidural analgesia and postoperative outcome. Lancet 2008;372:2109. author reply 2110.

18. Popping DM, Elia N, Marret E, et al. Protective effects of epidural analgesia on pulmonary complications after abdominal and thoracic surgery: A meta-analysis. Arch Surg 2008;143:990. discussion 1000 .

19. Wijeysundera DN, Beattie WS, Austin PC, et al. Epidural anaesthesia and survival after intermediate-to-high risk noncardiac surgery: A population-based cohort study. Lancet 2008;372:562.

20. Marret E, Remy C, Bonnet F. Meta-analysis of epidural analgesia versus parenteral opioid analgesia after colorectal surgery. Br J Surg 2007;94:665.

21. Werawatganon T, Charuluxanun S. Patient controlled intravenous opioid analgesia versus continuous epidural analgesia for pain after intra-abdominal surgery. Cochrane Database Syst Rev 2005;1:CD004088.

22. Holte K, Foss NB, Andersen J, et al. Liberal or restrictive fluid administration in fast-track colonic surgery: A randomized, double-blind study. Br J Anaesth 2007;99:500.

23. Lobo DN, Macafee DA, Allison SP. How perioperative fluid balance influences postoperative outcomes. Best Pract Res Clin Anaesthesiol 2006;20:439.

24. Macafee DA, Allison SP, Lobo DN. Some interactions between gastrointestinal function and fluid and electrolyte homeostasis. Curr Opin Clin Nutr Metab Care 2005;8:197.

25. MacKay G, Fearon K, McConnachie A, et al. Randomized clinical trial of the effect of postoperative intravenous fluid restriction on recovery after elective colorectal surgery. Br J Surg 2006; 93:1469.
26. Marjanovic G, Villain C, Juettner E, et al. Impact of different crystalloid volume regimes on intestinal anastomotic stability. Ann Surg 2009;249:181.

27. Muller S, Zalunardo MP, Hubner M, et al. A fast-track program reduces complications and length of hospital stay after open colonic surgery. Gastroenterology 2009;136:842.

28. Miller RD, Ed. Miller's anesthesia. Philadelphia: Elsevier Churchill Livingstone, 2005.

29. Bellomo R, Ronco C, Kellum JA, et al. Acute renal failure Definition, outcome measures, animal models, fluid therapy and information technology needs: The Second International Consensus Conference of the Acute Dialysis Quality Initiative (ADQI). Group Crit Care 2004;8:R204.

30. Dindo D, Demartines N, Clavien PA. Classification of surgical complications: A new proposal with evaluation in a cohort of 6336 patients and results of a survey. Ann Surg 2004;240:205.

31. de Aguilar-Nascimento JE, Diniz BN, do Carmo AV, et al. Clinical benefits after the implementation of a protocol of restricted perioperative intravenous crystalloid fluids in major abdominal operations. World J Surg 2009;33:925.

32. Nisanevich V, Felsenstein I, Almogy G, et al. Effect of intraoperative fluid management on outcome after intraabdominal surgery. Anesthesiology 2005;103:25.

33. Holte K, Jensen P, Kehlet H. Physiologic effects of intravenous fluid administration in healthy volunteers. Anesth Analg 2003; 96:1504.

34. Noblett SE, Snowden CP, Shenton BK, et al. Randomized clinical trial assessing the effect of Doppler-optimized fluid management on outcome after elective colorectal resection. Br J Surg 2006;93:1069.

35. Awad S, Allison SP, Lobo DN. Fluid and electrolyte balance: The impact of goal directed teaching. Clin Nutr 2008;27:473.

36. Basse L, Werner M, Kehlet H. Is urinary drainage necessary during continuous epidural analgesia after colonic resection? Reg Anesth Pain Med 2000;25:498. 